\title{
Sharing Receptive Fields with Your Neighbors: Tuning the Vertical System Cells to Wide Field Motion
}

\author{
Karl Farrow, Alexander Borst, and Juergen Haag \\ Department of Systems and Computational Neurobiology, Max-Planck-Institute of Neurobiology, 82152 Martinsried, Germany
}

\begin{abstract}
In the blowfly, the direction-selective response of the 60 lobula-plate tangential cells has been ascribed to the integration of local motion information across their extensive dendritic trees. Because the lobula plate is organized retinotopically, the receptive fields of the tangential cells ought to be determined by their dendritic architecture. However, this appears not always to be the case. One compelling example is the exceptionally wide receptive fields of the vertical system (VS) tangential cells. Using dual-intracellular recordings, Haag and Borst (2004) found VS cells to be mutually coupled in such a way that each VS cell is connected exclusively to its immediate neighbors. This coupling may form the basis of the broad receptive fields of VS cells. Here, we tested this hypothesis directly by photoablating individual VS cells. The receptive field width of VS cells indeed narrowed after the ablation of single VS cells, specifically depending on whether the receptive field of the ablated cell was more frontal or more posterior to the recorded cell. In particular, the responses changed as if the neuron lost access to visual information from the ablated neuron and those VS cells more distal than it from the recorded neuron. These experiments provide strong evidence that the lateral connections among VS cells are a crucial component in the mechanism underlying their complex receptive fields, augmenting the direct columnar input to their dendrites.
\end{abstract}

Key words: insect; blowfly; laser ablation; motion detection; neural network; receptive field

\section{Introduction}

In blowflies, the processing of large field motion is performed in the posterior division of the third neuropile of the optic lobe, the lobula plate. Within the lobula plate, there exist $\sim 60$ individually identifiable motion-sensitive neurons (tangential cells) per hemisphere. Among them, the vertical system (VS) cells form one of the major output elements. The direction-selective response to motion of tangential cells has been ascribed to the dendritic integration of local motion detector input onto the dendritic tree of each cell (Borst and Egelhaaf, 1992; Haag et al., 1992, 2004; Single and Borst, 1998). Because the input to the lobula plate is organized into retinotopic columns, the expected receptive field of each cell should be a consequence of the preferred direction of its local motion input, as well as its dendritic location and architecture.

The $10 \mathrm{VS}$ cells are typically T-shaped, with their overlapping dendritic arborizations lying serially, collectively covering the lobula plate. Their axons project centrally and run close together (Haag and Borst, 2004). They are sequentially named (VS1VS10), in which the dendrites of the VS1 cell reach the lateral edge of the lobula plate and the dendritic domains of successive neurons shift medially in accordance with their name. VS cells respond maximally to downward motion presented at a particular

\footnotetext{
Received Jan. 13, 2005; revised Feb. 28, 2005; accepted March 5, 2005.

This work was supported by the Max Planck Society. We thank Y. Choe for carefully reading this manuscript as well as D. Reiff and Y. Elada for helpful discussions.

Correspondence should be addressed to Karl Farrow, Department of Systems and Computational Neurobiology, Max-Planck-Institute of Neurobiology, Am Klopferspitz 18a, 82152 Martinsried, Germany. E-mail: kfarrow@neuro. mpg.de.

DOI:10.1523/JNEUROSCI.0168-05.2005

Copyright $\odot 2005$ Society for Neuroscience $\quad$ 0270-6474/05/253985-09\$15.00/0
}

position with a graded depolarization (Hengstenberg, 1977, 1982; Krapp et al., 1998). Based on the retinotopic input to the lobula plate and the area of the lobula plate covered by the dendrites of each cell (12-29\% for VS2-VS9) (Hengstenberg et al., 1982 ), the width of the receptive field should amount to $\sim 30-$ $40^{\circ}$. However, the receptive fields of VS cells are more complex than thought previously. One interesting characteristic is that VS cells respond to downward motion across a much wider slice of the visual world than expected by their dendritic extent (Krapp et al., 1998). In addition, the medial VS cells (VS8-VS10) respond to upward vertical motion in the frontal visual field (Krapp et al., 1998). This is far outside the visual space expected from their dendritic coverage of the lobula plate.

A recent dual-intracellular recording study demonstrated that VS cells are electrically connected, putatively via their axons (Haag and Borst, 2004). The connection strength was found to decrease with increasing distance between the VS cells. This could be explained in the most parsimonious way by assuming that each VS cell is connected exclusively to its immediate neighbors, forming a row of cells with the VS1 and VS10 cells at opposite ends. To account for the sign reversal found between VS1 and VS8-VS10 cells in the same study, an additional inhibitory connection had been postulated linking the medial and lateral cells. This connection scheme was proposed to be responsible for the extremely wide receptive fields of VS cells and the responsiveness of the medial VS cells to frontal visual stimuli.

Here, single VS cells were ablated to address three questions raised by the current injection experiments of Haag and Borst (2004). First, is the coupling between VS cells responsible for their broad receptive fields? Second, are the connections between the VS cells accomplished in a chain-like manner, or does each VS 
cell make reciprocal connections with all others separately? Third, can the posited inhibitory loop input from the VS1 cell to the medial VS cells account for the sensitivity of the medial VS cells to vertical motion in the frontal visual field?

\section{Materials and Methods}

Animal preparation. Two- to 7 -d-old female blowflies (Calliphora vicina) were briefly anesthetized with $\mathrm{CO}_{2}$ and mounted ventral-side up with wax on a small plate of glass. The head capsule was opened from behind, and the trachea and air sacs that normally cover the lobula plate were removed. To minimize movements of the brain, the proboscis of the animal was stretched forward and waxed to its thorax. Also, the antennas were removed, the antennal muscles were cut, and the abdominal regions were waxed. This allowed for stable intracellular recordings of up to 45 min. After alignment of the fly with reference to their deep pseudopupil, it was mounted on a heavy recording table facing two stimulating monitors. The fly brain was viewed from behind through a fluorescence stereo microscope (model MZ FLIII; Leica, Bensheim, Germany).

Electrophysiological recording. For intracellular recordings, glass electrodes were pulled on a Flaming/Brown micropipette puller (model P-97; Sutter Instrument, Novato, CA), using thin-walled glass capillaries with an outer diameter of $1 \mathrm{~mm}$ (GC100TF-10; Clark Electromedical Instruments, Pangbourne, UK). The tip of the electrode was filled with either $10 \mathrm{~mm}$ Alexa Fluor 568 hydrazide (Alexa 568) (Molecular Probes, Eugene, OR) or 6-carboxy-fluorescein in $1 \mathrm{M} \mathrm{KAc}$ (fluorescein; Molecular Probes). Alexa 568 and 6-carboxy-fluorescein fluoresce as red and green, respectively, allowing us to identify more than one cell at a time. The shaft of the electrode was filled with $2 \mathrm{~m}$ KAc plus $0.5 \mathrm{M} \mathrm{KCl}$. The electrodes had resistances between 15 and $25 \mathrm{M} \Omega$. Recorded signals were amplified using an SEL10 amplifier (NPI Electronic, Tamm, Germany). All recordings were made either in the axon or in the primary dendrites of a neuron. The output signals of the amplifier were passed to a Pentium III personal computer (PC) via a 12-bit analog-to-digital converter (DAS1602; ComputerBoards, Middleboro, MA) at a sampling rate of $5 \mathrm{kHz}$.

Visual stimulation. Visual stimuli were presented on two Tektronix (Wilsonville, OR) cathode ray tube monitors (width, $10 \mathrm{~cm}$; height, 13 $\mathrm{cm})$. The front monitor was located at a distance of $9.5 \mathrm{~cm}$ from the fly, and the side monitor was $9 \mathrm{~cm}$ away. The centers of the screens were located at an elevation of $0^{\circ}$ in reference to the equator of the fly's eyes. As seen by the fly, the two monitors together had a horizontal angular extent of $143^{\circ}$, starting at $-30^{\circ}$ frontally and reaching to $113^{\circ}$ posteriorly. The screens had a vertical extent of $69^{\circ}$. The pattern consisted of a square wave grating with a spatial wavelength of $25^{\circ}$, produced by an image synthesizer (Picasso; Innisfree, Cambridge, MA) at a frame rate of $200 \mathrm{~Hz}$. The image synthesizer was controlled by a Pentium III PC via a DDA06 board (ComputerBoards). The pattern moved at a velocity of $42 \%$, corresponding to a temporal frequency of $1.7 \mathrm{~Hz}$. The pattern contrast was $95 \%$. The mean luminance of the pattern amounted to $12 \mathrm{~cd} / \mathrm{m}^{2}$. The stimulation and acquisition software was written in Delphi (Borland, Scotts Valley, CA).

While recording a single cell, the stimulus pattern was presented at 10 different horizontal positions. The stripes were $18.5^{\circ}$ wide on the front screen and $18^{\circ}$ wide on the side screen. At each location, the pattern moved vertically. This setup allowed us to robustly stimulate the VS1VS6 cells in their central receptive fields. In addition, we could test the response of VS7-VS10 cells in the frontal part of their receptive field; their receptive field centers were outside the range of our monitors.

Data analysis. VS cells respond with a shift in membrane potential. All responses of cells here were determined by taking an average of the steady-state portion of the response minus the baseline. The steady-state portion of the response was determined to begin $0.5 \mathrm{~s}$ after the start of the stimulus. All tests of significance between means were unpaired $t$ tests.

Laser ablation. The cell to be ablated was filled with a saturated solution of 6-carboxy-fluorescein, which becomes toxic to cells as it fluoresces, in $1 \mathrm{~m} \mathrm{KAc} \mathrm{(}-2$ to $-10 \mathrm{nA} ; 1-15 \mathrm{~min})$. The neuron to be recorded was filled with Alexa 568, which, unlike 6-carboxy-fluorescein, is not toxic to the neuron while it fluoresces (Farrow et al., 2003). Subsequently, a $30 \mathrm{~mW}$ blue laser (488 nm; model 163-E11; Spectra-Physics, Fremont, CA) was used to selectively kill the fluorescein-filled cell.

\section{Results}

VS cells respond to motion presented in front of the ipsilateral eye with a graded shift in membrane potential superimposed with high-frequency events that are attributable to either active currents or synaptic input. Active properties are present in each VS cell and produce spikelets that are particularly prominent in the VS1 cell (Hengstenberg, 1977, 1982; Haag et al., 1997), whereas EPSP activity in medial VS cells (VS7-VS10) is attributable to excitatory input, putatively from a spiking interneuron (Haag and Borst, 2004). In Figure $1 A$, a schematic of the receptive fields of a VS2, VS4, and VS6 cell is shown together with the connectivity among VS cells as determined via double intracellular recordings (Haag and Borst, 2004). Each VS cell has a receptive field center (Fig. $1 \mathrm{~A}$, black arrows) that is attributed to the integration of local motion information across its dendrites (Borst and Egelhaaf, 1992; Haag et al., 1992, 2004; Single and Borst, 1998). The location of the dendrites within the lobula plate corresponds to the position of the receptive field center of each cell: as one moves laterally in the lobula plate, the receptive fields of the cells shift frontally. In addition, each cell responds to motion stimuli outside its central receptive field (Krapp et al., 1998). For example, all of the VS cells respond to downward motion across a much broader slice of visual space than that predicted by the extent of their dendrites (Fig. $1 A$, gray arrows).

We marked each recorded VS cell by dye injection and identified it using a combination of its characteristic branching pattern in the lobula plate, as determined in previous neuroanatomic studies (Hengstenberg et al., 1982; Krapp et al., 1998) and measurements regarding the relative location of the ventral dendritic branch of each cell within the lobula plate (data not shown). The measurements of the location of ventral dendrites were made from pictures of fly brains $(n=8)$, in which a minimum of five cells were labeled. By combining the subjective anatomical information with a quantification of the location of the main ventral dendrite of each VS cell, we could unambiguously identify each filled VS cell.

In Figure $1 B$, sample traces of a VS2 and a VS4 cell recorded from the same fly are shown. Notice that both cells respond to downward motion with a depolarizing shift in membrane potential. However, the azimuthal stimulus position at which they are maximally responsive differs: although the maximum response of the VS2 cell is obtained at a horizontal position of $5^{\circ}$, the VS4 cell responds most strongly to pattern motion at $52^{\circ}$. In addition, both cells exhibit at least small responses at all stimulus locations.

The receptive fields of VS cells (VS1-VS6) were determined in response to thin upright stripes of horizontal grating moving vertically (Fig. $1 C$ ). Each data point is the normalized mean \pm SEM. The two most striking features of the receptive fields of the VS cells are their width and the amount of overlap between neighboring cells. In particular, the receptive fields of the VS1-VS3 cells are almost identical. Each of these three cells shows a strong response, $>33 \%$ of maximum, at positions ranging from -8 to $52^{\circ}$. In addition, significant responses can still be detected at the most posterior stimulus positions ( 91 and $104^{\circ}$ ). The VS4-VS6 cells also have highly overlapping receptive fields, although not to the same extent as those of the VS1-VS3 cells. The receptive field peaks of the VS4-VS6 cells are located, as expected from the location of the dendrite of each cells, at 52, 78, and $104^{\circ}$, respectively. However, these three cells have exceptionally wide receptive fields showing significant responses at every stimulus position, with responses $>33 \%$ of the peak spanning 112,99 , and $86^{\circ}$, respectively (Fig. 1C). Note that the stimulus device did not allow 
A

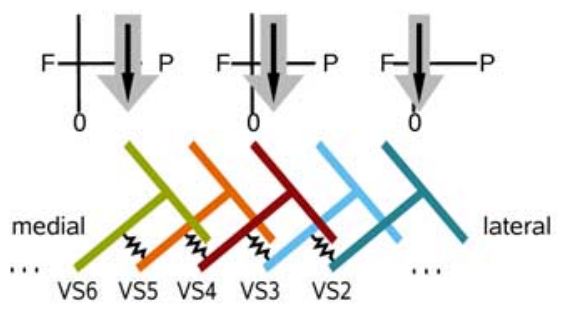

Electrical

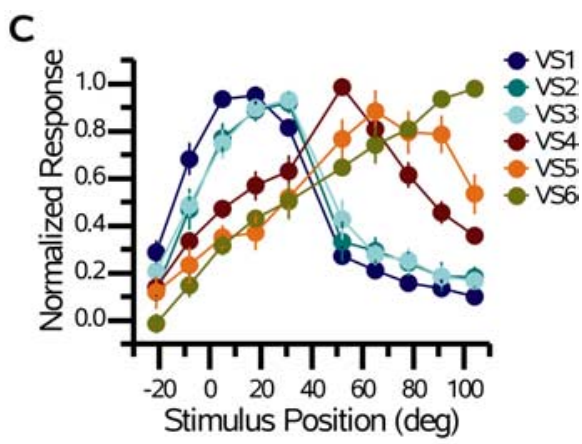

B

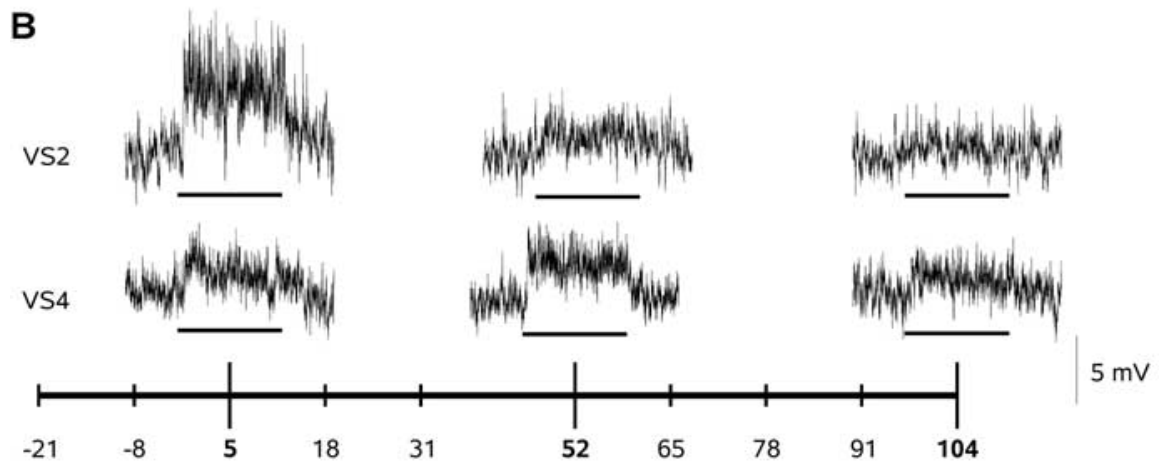

Stimulus Position (deg)

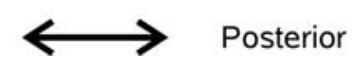

Figure 1. VS cell network and receptive fields. $A$, Schematic network of VS cells. Above the VS6, VS4, and VS2 cells is a diagram of the receptive field of each cell (gray and black arrows). The black arrows indicate the central receptive field of each cell, whereas the gray arrows show its spatial extent. As one moves laterally in the lobula plate, the receptive fields move frontally in visual space. $\boldsymbol{B}$, Single responses of a VS2 and VS4 cell recorded from the same fly show the basic response properties of VS cells. Note that both cells respond to downward motion with a graded shift in membrane potential. The response amplitude depends on the stimulus position (see arrows). For each trace, the stimulus was applied for $1 \mathrm{~s}$. The scale bar is relevant for each trace. $C$, Responses of six VS cells to downward motion as a function of stimulus position. Zero degrees on the $x$-axis represents the position directly in front of the fly, and positive numbers represent positions on the same side on which the cells were recorded. Each data point is the mean response $\pm S E M$, normalized with respect to its maximum. Note the strong overlap of VS1 $(n=12)$, VS2 $(n=7)$, and VS3 $(n=9)$ cells. In addition, the responses of the VS4 $(n=13)$, VS5 $(n=5)$, and VS6 $(n=6)$ cells shift posteriorly. deg, Degrees.

us to record the full extent of the receptive fields of these cells, because our most posterior stimulus was centered at $104^{\circ}$. Using a different stimulation design, unambiguous responses to downward motion have been noted in the VS4-VS6 cells across the entire ipsilateral visual field (Krapp et al., 1998). Another interesting feature of the receptive fields is the apparent grouping of the different VS cells. In particular, the separation between the receptive fields of the VS3 and VS4 cells is much greater than that between the receptive fields of VS2 and VS3 cells or VS4 and VS5 cells, respectively.

\section{Proximal versus distal ablations}

The broadening of the receptive field of each VS cell, beyond its central receptive fields, has been attributed to input from neighboring cells via electrical synapses in the axons (Haag and Borst, 2004). These electrical connections are thought to be solely between neighboring VS cells. According to this view, for example, if the VS5 cell is influenced by the input to the dendrites of the VS2 cell, this information must pass through both the VS3 and VS4 cells before affecting the activity of the VS5 cell. To determine whether the electrical coupling of neighboring VS cells does indeed affect the width and overlap between the receptive fields of the neighboring VS cell, we ablated single VS cells and examined the receptive field structure of a nearby cell. In each experiment, we first filled a single cell with fluorescein (Figs. 2, green cells, 4, 7). After withdrawing the electrode, we filled a second cell with Alexa 568 (Figs. 2, red cells, 4, 7). Subsequently, we recorded the response of this cell to downward motion at several positions, both before and after the ablation of the fluorescein-filled cell. Figure $2 A$ shows how the ablation of a VS2 cell affects the receptive field shape of a VS4 cell. Before ablating the VS2 cell, the VS4 cell responded strongly ( $\sim 50 \%$ of its peak response) to stimuli at those positions corresponding to the receptive field maxima of the VS2 cell (from 0 to $30^{\circ}$ ). After the ablation (of the VS2 cell), the response of the VS4 cell at the stimulus locations 5,18 , and $31^{\circ}$ dropped significantly $(p<0.05$; $t$ test) from $\sim 50$ to $\sim 15 \%$ of its peak response. This drop in response magnitude is also evident in the most frontal stimulus position, in which the response of the VS2 cell is only $\sim 10 \%$ of the peak (Fig. $2 A, C)$. In contrast, the relative response magnitude of the VS4 cell was maintained in the posterior stimulus positions, in which the VS2 cell is not highly responsive, and input to the VS4 cell can be both direct from local motion elements or via other VS cells with more posteriorly located receptive fields (Fig. 2C). This example implies that the VS2 cell supplies information to the VS4 cell about its own receptive field and that of the VS1 cell but not about cells with receptive fields more posterior than its own.

This association among nearby VS cells is not unique to the VS4-VS2 pair. Figure $2 D-F$ contains an example of a VS5 cell, both before and after the ablation of a VS6 cell. Here, the ablated cell had a more posterior receptive field. Consequently, when the VS6 cell was ablated, the VS5 cell experienced a decline of sensitivity in the posterior, but not in the frontal, divisions of its receptive field (Fig. 2D,F). A third example (Fig. 2G-I) clearly demonstrates how one cell can act as a conduit for passing information not only about its own activity but also about that of other cells farther away. Figure $2 G$ shows the receptive field of a VS1 cell both before and after the ablation of a VS3 cell. As in the previous example, the sensitivity in the receptive field of the VS1 cell fell in the posterior parts of its receptive field, albeit to a lesser degree (Fig. 2G,I). Nevertheless, the percentage change was large and significantly decreased $(p<$ 0.05 ) at each stimulus position posterior to the peak response of the VS3 cell, thus demonstrating that the input to the VS1 cell about these posterior stimulus positions is relayed via the VS3 cell.

To indicate the reproducibility of the effect of ablating single VS cells on the receptive fields of nearby neurons, we grouped four experiments of VS4 cells in which one of two more frontally viewing VS cells, the VS2 or VS3 cells, was ablated (Fig. 3). Figure 3 shows both the absolute (Fig. 3B) and relative (Fig. 3C) differ- 
A

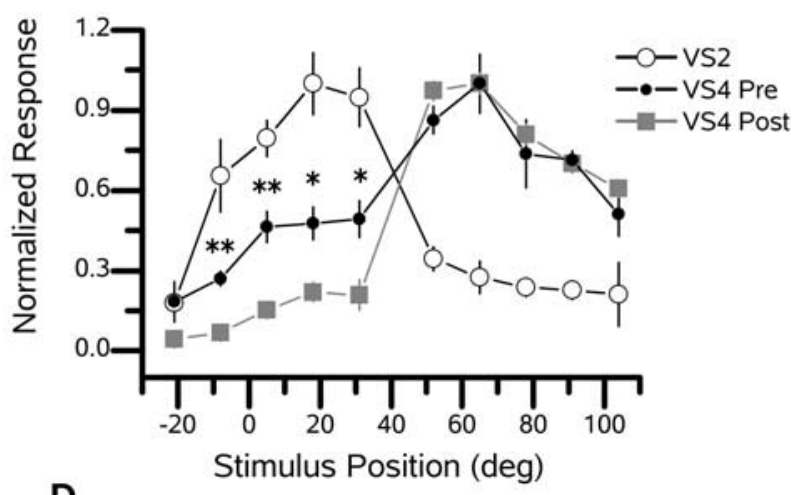

D

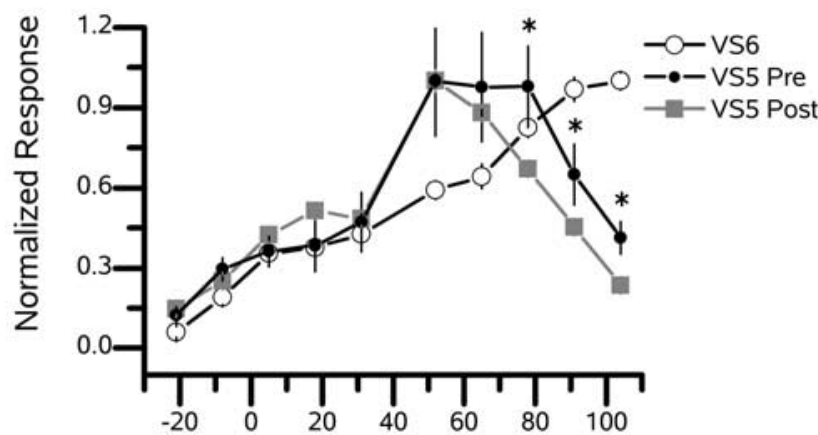

Stimulus Position (deg)

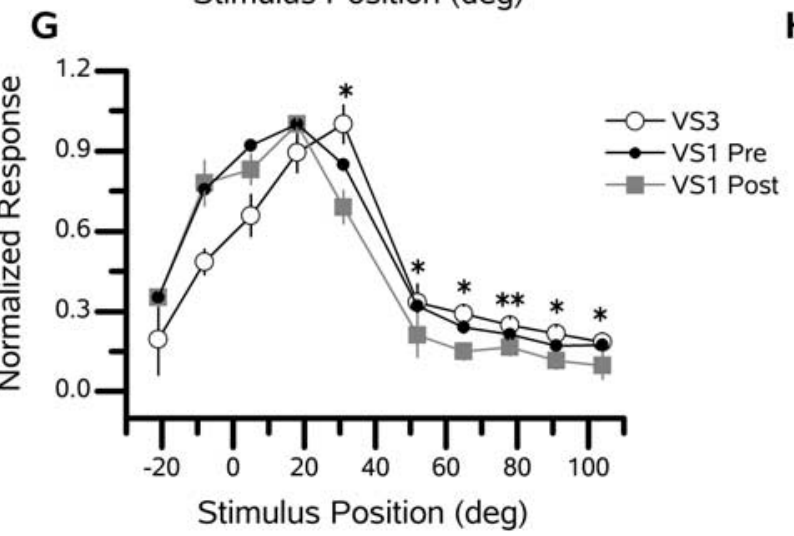

B

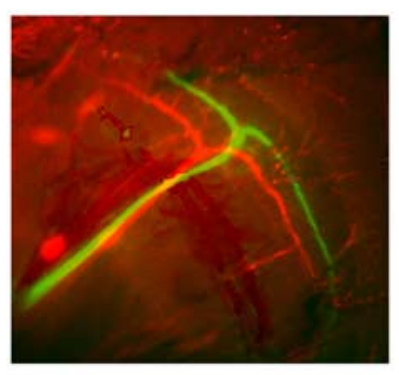

E

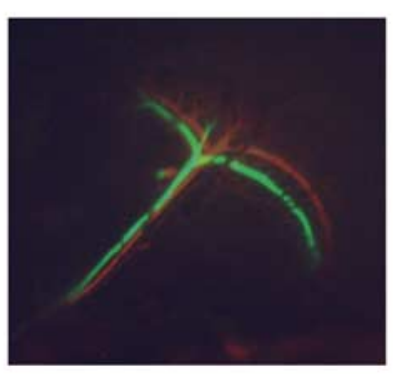

H

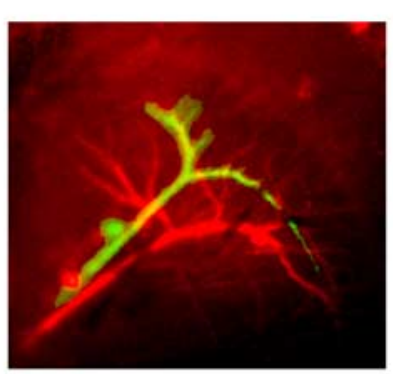

C

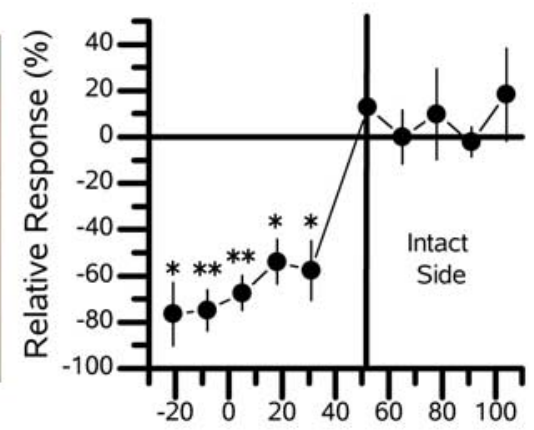

F

Stimulus Position (deg)

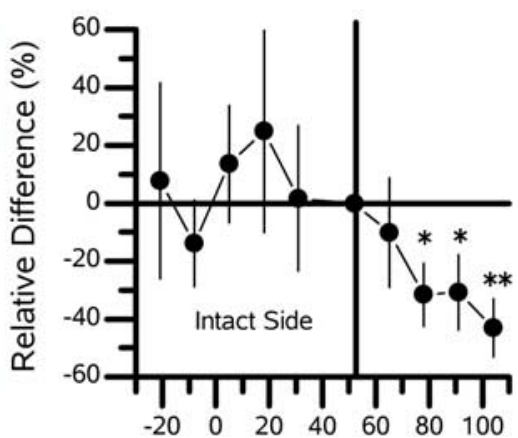

Stimulus Position (deg)

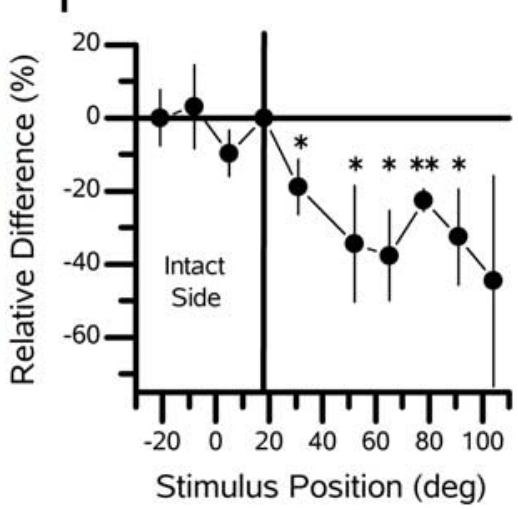

Figure 2. Proximal cell ablations. Three examples of the effect of ablating individual VS cells ( $B, E, H$, green cells) on the receptive field of a neighbor or next-of-neighbor VS cell ( $B, E, H$, red cells) are shown. In $A, D$, and $G$, the open circles connected with the blue line indicate the receptive field of the ablated cell (green cell), the filled orange circles indicate the receptive field of the VS cell in the intact animal (red cell), and the dark red filled squares indicate the receptive field after the green cell has been ablated. The $x$-axis shows the horizontal position at which the stimulus was applied. The receptive field for each set of recordings was normalized to the maximum response. Asterisks indicate positions at which significant changes between the preresponses versus postresponses of the recorded VS cell occurred $\left({ }^{*} p<0.05\right.$; $\left.{ }^{* *} p<0.001\right)$. In $\boldsymbol{C}, \boldsymbol{F}$, and $\boldsymbol{I}$, the relative difference [(post - pre)/pre $\times 100$; in percentage] between the preresponses (orange) and the postresponses (dark red) at each stimulus location is shown. The vertical line indicates the stimulus position at which the intact (red) cell had its peak response. $A$, An example of the receptive field of a VS4 cell before (Pre) and after (Post) the ablation of a VS2 cell. The peak response of the VS2 cell was $2.2 \mathrm{mV}$. The peak response of the VS4 cell was $1.7 \mathrm{mV}$ before and $2.4 \mathrm{mV}$ after the ablation of the VS2 cell. $\boldsymbol{B}$, VS4 and VS2 cells. C, Relative difference (percentage) of the preresponse versus the postresponse of the VS4 cell after the ablation of the VS2 cell. $\boldsymbol{D}$, A second example of a neighbor-neighbor ablation, showing the deficit a VS5 (red) cell experiences after the ablation of a VS6 (green) cell. The peak response of the VS6 cell was $9.0 \mathrm{mV}$. The response of the VS5 cell was $4.6 \mathrm{mV}$ before and $3.6 \mathrm{mV}$ after the ablation of the VS5 cell. $\boldsymbol{E}$, VS6 and VS5 cells. $\boldsymbol{F}$, Relative difference (percentage) of the preresponse versus the postresponse of the VS5 cell after the ablation of the VS6 cell. G, A third example of a neighboring cell ablation, demonstrating the change in a VS1 (red) cell after the ablation of a VS3 (green) cell. The peak response of the VS3 cell was $4.4 \mathrm{mV}$. The peak response of the VS1 cell was $7.7 \mathrm{mV}$ before and $5.6 \mathrm{mV}$ after the ablation of the VS3 cell. $\boldsymbol{H}$, VS1 and VS3 cells. $\boldsymbol{I}$, Relative difference (percentage) of preresponse versus postresponse of the VS1 cell after the ablation of the VS3 cell. deg, Degrees.

ence in the responses of the VS4 cells after the ablation of single $\operatorname{VS} 2(n=1)$ or VS3 $(n=3)$ cells. Each data point is the mean difference $( \pm$ SEM) between the normalized response after the ablation and the normalized response in the intact animal. The response of the VS4 cells at the position at which the responses of the VS2 and VS 3 cells are largest, for stimulation at 5, 18, and $31^{\circ}$, dropped by 0.225 (Fig. $3 B$ ), which is a relative difference of $-46 \%$ (Fig. 3C). The mean relative difference of the field for the two most frontal stimuli positions, -5 and $-21^{\circ}$, amounted to $-48 \%$ and, thus, was almost identical to that of the other three frontal stimulus positions. These two stimulus positions are located more frontally than the peak of the receptive fields of the VS2 and VS3 cells and indicate that the input from more frontal viewing cells is also interrupted when a single VS cell is ablated. This finding is in contrast to the change in the response of the VS4 cells in the more posterior stimulus position. Here, the response of the 

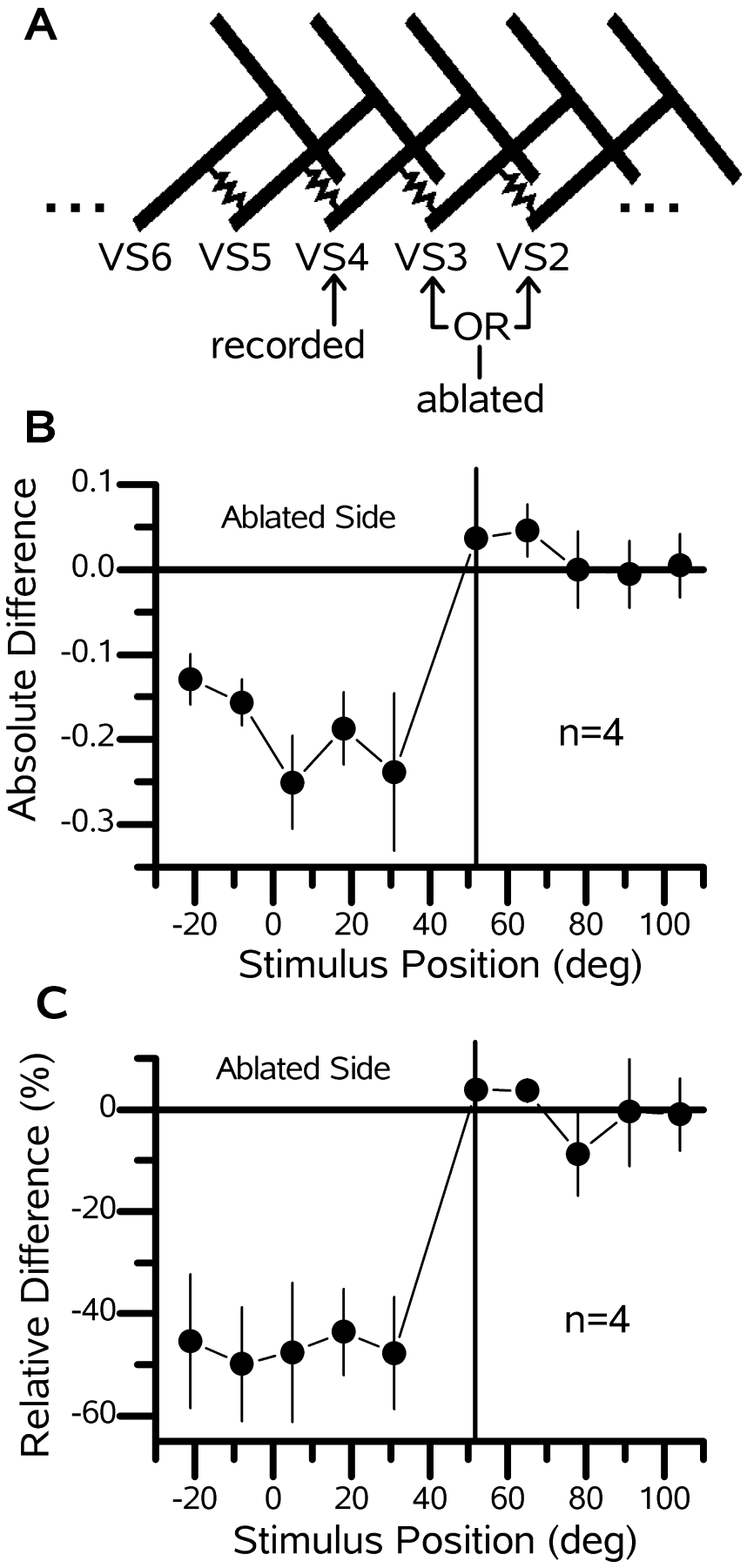

Figure 3. Deficit of a VS4 cell after the ablation of frontal viewing VS cells. A, Schematic of the VS cell network showing the relationship between the recorded cell (VS4) and the ablated cells (VS2 or VS3). B, Mean difference \pm SEM of the receptive fields (postresponse minus preresponse) for a group of four VS4 cells in which either a VS2 $(n=1)$ or a VS3 $(n=3)$ cell was ablated. $C$, Relative difference [(post - pre)/pre $\times 100$; in percentage] for the data shown in $\boldsymbol{B}$. Note that, at each stimulus location frontal to the peak response of the VS4 cell, the response drops by approximately the same amount ( $\sim 50 \%)$. deg, Degrees.

VS4 cells did not change at all. These results show that the VS4 cell inherits the frontal part of its receptive field from VS2 and VS3 cells and also imply that the VS1 cell plays a role.

To determine whether cells with respective receptive field peaks that are widely separated interact, we performed experiments in which the cell pairs were between four and six cells apart. An example of a VS6 cell with a VS1 cell ablated demon-
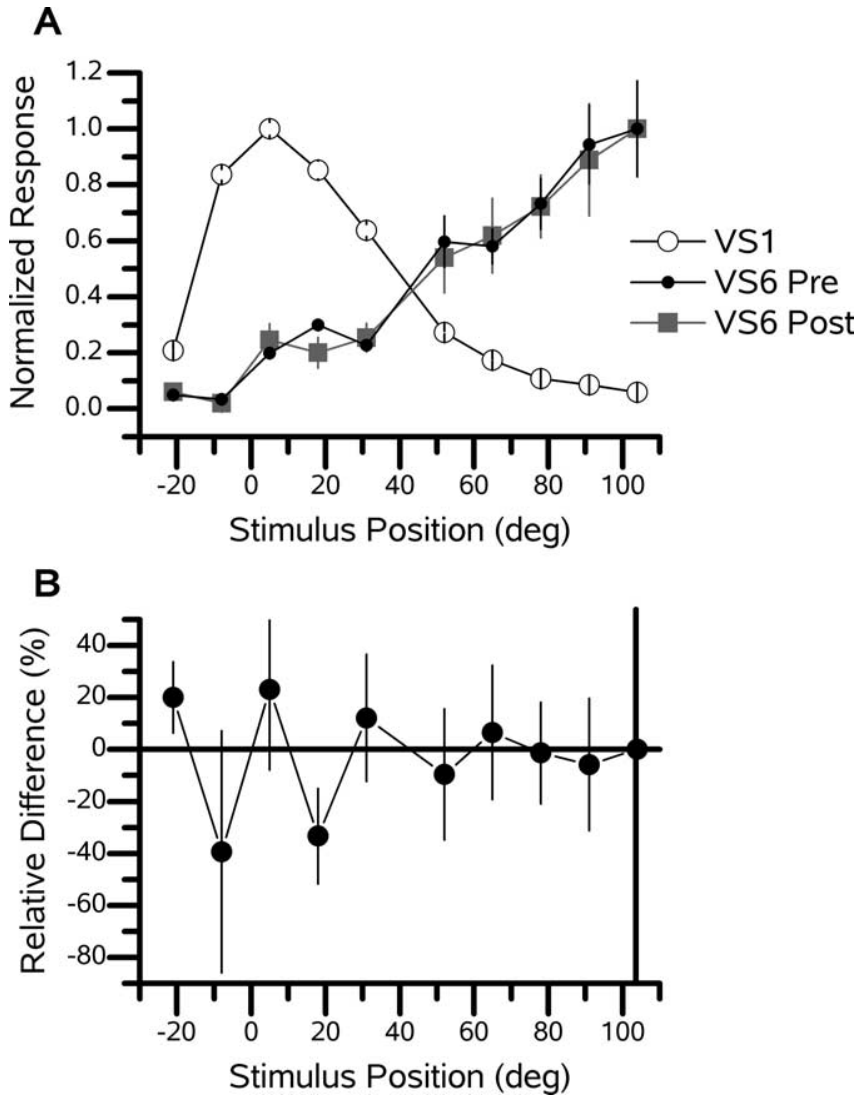

Figure 4. Distal ablation. An example of a VS6 cell after the ablation of a distant VS1 cell is shown. $A$, Receptive field of a VS6 cell before (Pre) and after (Post) the ablation of a VS1 cell. The peak response of the VS1 cell was $8.2 \mathrm{mV}$. The peak response of the VS6 cell was $1.6 \mathrm{mV}$ before and $1.7 \mathrm{mV}$ after the ablation of the VS1 cell. $\boldsymbol{B}$, Relative difference (percentage) of the response of the VS6 cell before versus after ablation.

strates that cells far apart do not influence each other (Fig. 4). After the VS1 cell was ablated, there was no detectable change in the receptive field structure of the VS6 cell. Similar results were obtained with a VS3-VS7 pair (data not shown). These two examples illustrate that cells between four and six cells apart do not affect each other's response.

A summary of all ablations performed is shown in Figure 5. The mean relative change for the intact versus the ablated side is plotted for each individual experiment (Fig. 5A). We define the ablated side to include all azimuthal stimulus positions on the same side of the receptive field peak of the recorded cell as the ablated cell, not including the position of the peak response. The intact side consists of the location of the peak response and those on the opposite side of the peak from the ablated cell. Note that for each experiment in which a neighboring (or neighboring but one) cell was ablated, the response on the ablated side dropped, whereas it remained the same on the intact side (Fig. $5 A$, red data points).

Because of concerns that cell ablations might alter the primary visual response of VS cells, we compared the peak response amplitude and the stimulus position at which this peak occurs for each cell (Fig. $5 B, C$ ). Before and after ablations, we found no consistent effect of ablating a single neuron on the response of neighboring cells. The mean peak response for all experiments fell from 3.9 to $3.3 \mathrm{mV}$, which was not significant $(n=12 ; p=$ 0.42 ). For the set of eight ablations in which a neighbor or nextof-neighbor cell was ablated, the peak response also did not change. In addition, the stimulus position of the peak response 


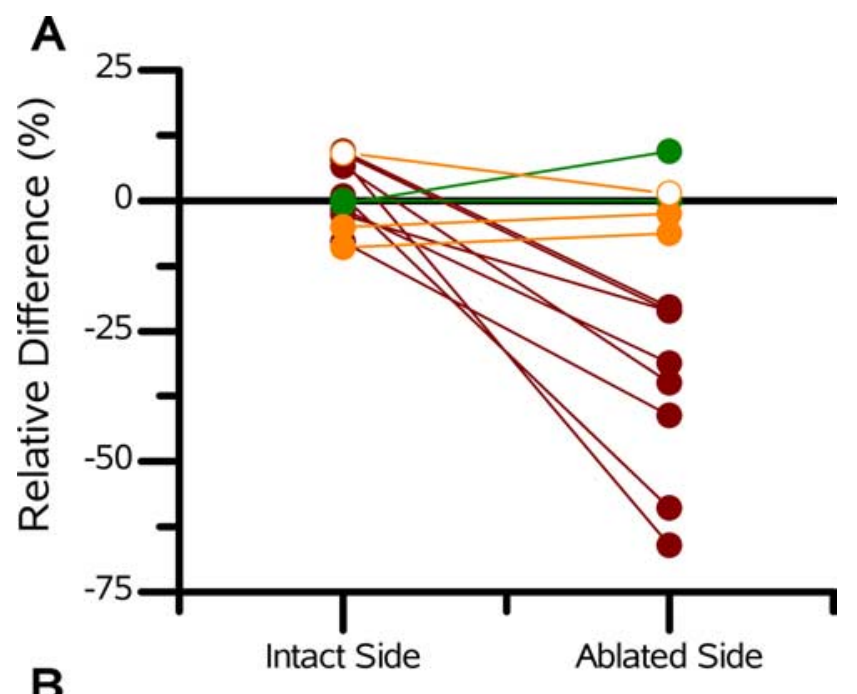

B

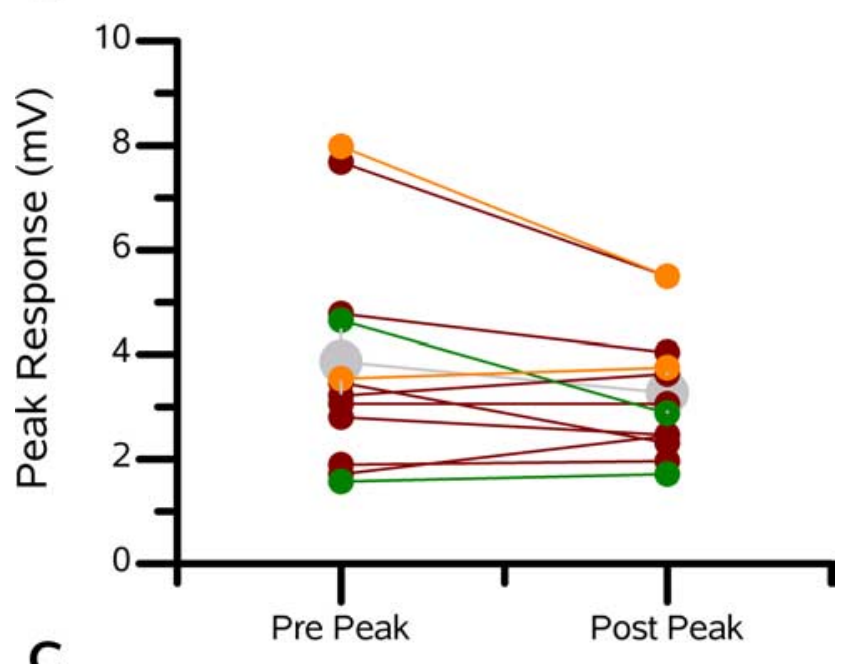

C

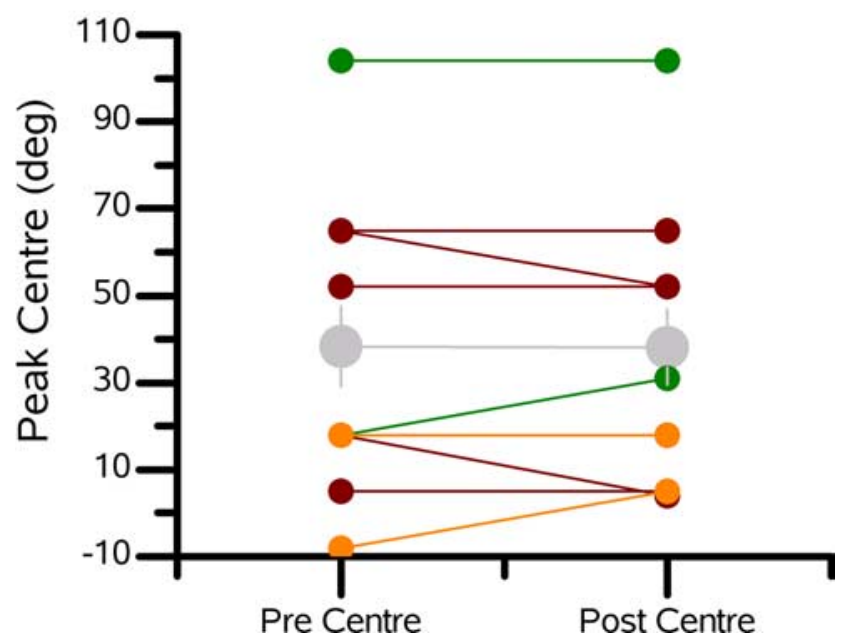

Figure 5. Summary of ablations. $\boldsymbol{A}$, Mean relative difference between the postablation and preablation responses shown for both the ablated side and intact side of the receptive fields of the recorded VS cells. The ablated side consists of those stimulus positions at and beyond, from the point of view of the recorded cell, the peak of the ablated cell. The intact side comprises all other stimulus positions. In each graph, the red data points represent the neighbor ablations, the green data points represent the distant ablations, and the orange data points represent VS1 cell ablations while recording one of the medial VS cells. $\boldsymbol{B}$, Peak responses for each experiment before and after the ablation of a single VS cell. The gray data points represent the mean \pm SEM of all experiments. $\boldsymbol{C}$, Peak response position for each cell before and after the ablation of another VS cell. The gray data points represent the mean \pm SEM. deg, Degrees. remained stable. These results suggest that the observed narrowing of the receptive fields is not attributable to damage done to the input from the local motion detectors but rather to deficits in the input from neighboring VS cells.

\section{VS1 cell input to the medial VS cells}

In addition to possessing the above-mentioned broad receptive fields, medial VS cells (VS8-VS10) respond unexpectedly to vertical motion presented in the frontal visual field, far from their presumed local motion input (Krapp et al., 1998). Current injection into VS1 cells influenced the activity of the medial VS cells (Haag and Borst, 2004), and this connection has been proposed to underlie the sensitivity of the medial VS cells to motion in the frontal visual field (Fig. 6A). In line with this proposal (Haag and Borst, 2004), the receptive fields of the medial VS cells (VS8VS10) and the VS1 cell have opposite polarity (Fig. 6B): upward motion in the frontal visual field hyperpolarizes the VS1 cell, but the same stimulus depolarizes the VS8-VS10 cells. In addition, the widths of the receptive fields of the medial VS cells in response to upward motion overlap, which is consistent with a common input. Each cell produced a response of $>33 \%$ spanning a minimum of $50^{\circ}$ ranging from -21 to $31^{\circ}$.

To determine whether inhibitory input to the medial VS cells from the VS1 cell can account for their sensitivity to vertical motion in the frontal visual field, we recorded the receptive fields of single medial VS cells before and after the ablation of the VS1 cell. In Figure 7, the receptive field to upward (Fig. 7A) and downward (Fig. 7D) motion of a VS8 cell (Fig. 7C, red cell) is shown before and after the ablation of a VS1 cell (Fig. 7C, green cell). No significant differences were found after the ablation of the VS1 cell (Fig. $7 B, G$ ). This was also true for another ablation experiment involving the response of a VS9 cell to upward motion before and after the ablation of a VS1 cell. Here, unlike in the proximal and distal ablations, the ablated side included the five most frontal stimulus positions, whereas the intact side included the five most posterior stimulus positions. The grouped average response magnitude of the five frontal and five posterior stimulus positions did not change significantly after the ablation of a VS1 cell (Fig. 5A, orange points).

In contrast to our expectations, these experiments show that input from the VS1 cell cannot be solely responsible for the sensitivity of the three medial VS cells to vertical motion in the frontal divisions of their receptive fields.

\section{Discussion}

VS cells respond to stimuli presented outside of the visual space expected from the retinotopy of the lobula plate and the extent of their dendritic arborization. The receptive fields are unexpectedly wide, and, in the case of the medial VS cells (VS8-VS10), include frontal sensitivity when only posterior vision was expected (Krapp et al., 1998). The results of our ablation experiments show that lateral connections among VS cells form the basis of their unexpectedly wide receptive fields, which substantially exceed the proportion of the lobula plate covered by their dendrites. We also provide evidence that VS cells connect sequentially in a chain-like manner beginning with the VS1 cell on the lateral edge of the lobula plate and continuing through each VS cell to the VS10 cell on the medial edge of the lobula plate. However, how the medial VS cells acquire sensitivity in the frontal visual field remains unclear.

\section{VS cell network}

The serial connection scheme outlined above was suggested by Haag and Borst (2004) based on the magnitude and bidirection- 
A

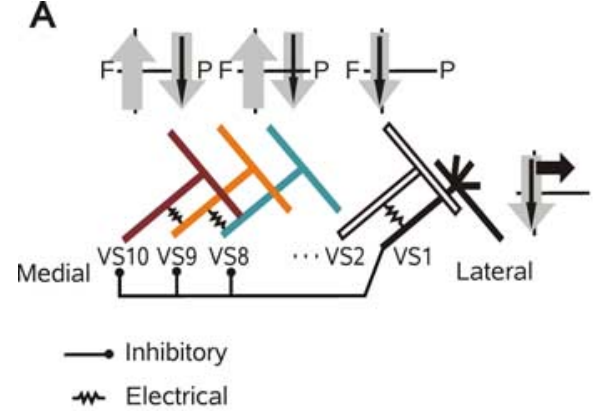

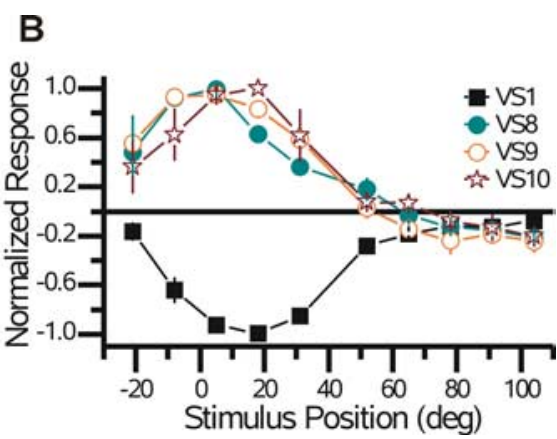

Figure 6. VS1 cell receptive fields. A, Schematic network of VS cells highlights the hypotheses of Haag and Borst (2004). It is unclear to which of the medial VS cells the VS1 cell provides inhibitory input. The receptive fields of the VS2, VS8, and VS10 cells are shown above the respective cell. The format is the same as that in Figure $1 A$. F, Frontal; $P$, posterior. $\boldsymbol{B}$, The receptive fields of the VS1 cell and the three most medial, posterior viewing VS cells in response to upward motion. See Figure $1 B$ for explanation. Note the overlap among the VS8 $(n=2)$, VS9 $(n=3)$, and VS10 $(n=2)$ cells. In addition, the VS1 cell appears to mirror that of the medial VS $(n=5)$ cells. deg, Degrees.
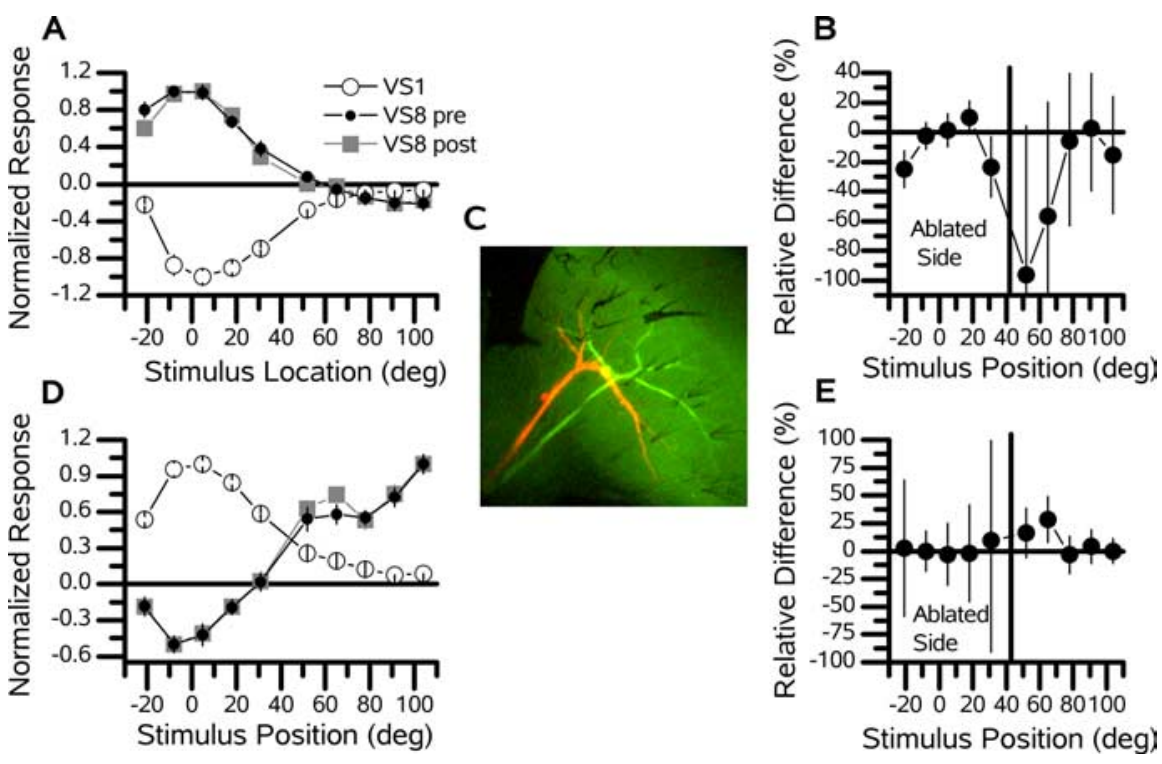

Figure 7. VS1 cell ablation. Example recordings of a VS8 (red) cell before and after the ablation of a VS1 (green) cell. The vertical line separates the five frontal stimulus positions (the ablated side) from the five lateral stimulus positions (the intact side). $\boldsymbol{A}$ Response of a VS8 cell to upward motion before (pre) and after (post) the ablation of a VS1 cell. The peak response of the VS1 cell was $-6.6 \mathrm{mV}$, whereas that of the VS 8 cell was $3.5 \mathrm{mV}$ before and $3.7 \mathrm{mV}$ after the ablation of the VS1 cell. The peak response of the VS 8 cell was in its frontal receptive field. $\boldsymbol{B}$, Relative difference (percentage) of the preresponse versus the postresponse of the VS8 cell to upward motion. C, Two recorded cells. D, Response of the same VS8 cell to downward motion before and after the ablation of a VS1 cell. The peak response of the VS1 cell was $14.5 \mathrm{mV}$, whereas that of the VS 8 cell was normalized to $2.6 \mathrm{mV}$ before and $2.1 \mathrm{mV}$ after the ablation of the VS1 cell. The peak response of the VS8 cell was at the most posterior stimulus position. $\boldsymbol{E}$, Relative difference (percentage) of the preresponse versus postresponse of the VS8 cell to downward motion. deg, Degrees.

ality and temporal properties of the signals passing between individual VS cells. However, the results of these current injection experiments did not exclude the possibility that the VS cells make reciprocal contact to the VS1 cell individually with disparate properties. Our experiments provide additional evidence that the VS cells are connected in the proposed chain-like manner and suggest that this connectivity scheme generalizes to all VS cells.

Here, after the ablation of a single VS cell, the recorded VS cell had reduced responses to vertical motion in specific locations of their receptive field dependent on the location of the ablated cell. If the VS cells were fully interconnected such that each cell made reciprocal recurrent connections with all other VS cells, the response deficit would only be expected to occur at stimulus positions in which the peak response of the ablated cell was located, causing a dip in the receptive field of the recorded VS cell. However, the responses of the recorded VS cell fell significantly not only at the stimulus positions at which the ablated cell was most responsive, but also at stimulus locations farther away than the peak responses of the ablated cell (Fig. $2 A, G)$. This caused a flattening of the receptive field of the recorded cell toward zero that was dependent on the location of the ablated cell. This was shown not just for the VS1-VSx pairs (any pair of VS cells, including the VS1 cell) but for a range of cell pairs including the following: VS1VS2, VS1-VS3, VS2-VS1, VS4-VS2, VS4-VS3, and VS5-VS6 (recorded-ablated cell). This implies that ablating a single cell breaks the chain of VS cells, stopping the flow of information to the recorded cell from neurons beyond the ablated cell.

\section{Residual responses}

One caveat of our results is that the responses of the VS cells to stimuli putatively outside of their dendritic receptive field were not completely abolished. The mean relative change after the ablation of a single VS cell dropped a maximum of $66 \%$ and a minimum of $21 \%$ (Fig. $5 A$ ). One explanation for this involves the limitations of our ablation technique. It is not exactly known whether strong illumination ablates a dyefilled cell as a whole or only a part of it. Generally, photoablation causes the resting membrane potential of the cell to depolarize to zero and causes the input resistance of the neuron to vanish (Farrow et al., 2003). However, it has been shown that the selective partial ablation of a dye-filled cell is possible with a focused laser beam (Miller and Selverston, 1979; Jacobs and Miller, 1985). Although our laser was not focused and illuminated the entire lobula plate, it is still conceivable that laser illumination ablated large parts of the VS cells but left electrically intact those segments in which VS cells make contact. In such a case, although the retinotopic input from the ablated cell would be abolished, the remaining segments could still function as a relay for input from cells farther away. Alternatively, if these cells, in addition to being coupled to their neighbors, make common downstream connections via electrical synapses, some of the residual responses could be attributable to the passage of current via these output targets.

\section{VS1 cell ablation: why no effect?}

After the ablation of the VS1 cell, we found that the response of the medial VS cells to upward and downward motion was unchanged (Figs. $5 A, 7 A, D$ ). This result came as a surprise, because it has been demonstrated previously that current injections into VS1 cells affect the activity of the medial VS cells (Haag and Borst, 2004). There are two explanations for this apparent contradic- 
tion. The first involves the possible connectivity among the VS cells. Perhaps all three frontally viewing VS cells provide inhibitory input to the medial VS cells independently. This would allow the VS2 and VS3 cells to compensate for the loss of the VS1 cell. This is plausible because these cells have highly overlapping receptive fields and have been shown previously to have a common output target, the V1 cell (Kurtz et al., 2001; Haag and Borst, 2003; Warzecha et al., 2003).

The second explanation is the inconsistency between the current injections performed by Haag and Borst (2004) and the response properties of the medial VS cells recorded here. Haag and Borst (2004) showed that positive, but not negative, current is passed from the VS1 to the medial VS cells. In addition, the medial VS cells have been reported to be selective to upward motion in the frontal visual field without an indication as to whether they depolarized or hyperpolarized in response to a particular direction of motion (Krapp et al., 1998). These two results are consistent if, during downward motion, the medial VS cells hyperpolarized, whereas during upward motion, the medial VS cells remained at rest. However, here we find that the medial VS cells do depolarize and hyperpolarize in response to upward and downward motion, respectively (Figs. 6B, 7A,D). Therefore, after the ablation of the VS1 cell, one would not expect a change in its response to upward motion, because, during such a stimulus, the VS1 cell hyperpolarizes, and thus its signal would not be passed on to the medial VS cells. The results here suggest that the experiments of Haag and Borst (2004) did not reveal the complete circuitry responsible for the sensitivity of the medial VS cells to motion in the frontal receptive field.

\section{Why the broad receptive fields?}

What is the advantage for VS cells to import the receptive fields of their neighbors via electrical synapses? Intuitively, this appears to make the output of individual neurons more ambiguous with respect to the location of the stimulus within the visual space and consequently more difficult for downstream neurons to extract useful information. However, this decrease in spatial resolution should be accompanied by a reduction in stochastic noise. Electrical connections among homologous cells that act to reduce noise as a result of stochastic events have been described at many visual processing stages. Examples include the cones (Raviola and Gilula, 1973; Kolb and Jones, 1985; Owen, 1985; Tsukamoto et al., 1992), amacrine cells (Famiglietti and Kolb, 1975; Vaney, 1991; Stettoi et al., 1992; Feigenspan et al., 2001), and ganglion cells (Vaney, 1991; Hidaka et al., 2004) in the vertebrate retina as well as the photoreceptors (Ribi, 1978) and lobula-plate tangential cells (Haag and Borst, 2002, 2004) of insects.

The effectiveness of lateral connection to aid noise reduction has been clearly demonstrated for cone photoreceptors that are electrically coupled, without greatly affecting visual signal acuity (Lamb and Simon, 1976; Tessier-Lavigne and Attwell, 1988; DeVries et al., 2002). Similarly, in the inner retina, electrical coupling among AII amacrine cells helps improve signal-to-noise ratios (Bloomfield and Völgyi, 2004). Interestingly, the electrical coupling between AII amacrine cells is 10 times larger during twilight-like light conditions (low signal-to-noise ratio) than under bright light conditions, which corresponds with the receptive field sizes of these cells under the same condition (Bloomfield and Völgyi, 2004).

The response to motion of lobula-plate tangential cells is also degraded by stochastic noise (Laughlin et al., 1987; de Ruyter van Steveninck and Bialek, 1995; de Ruyter van Steveninck and Laughlin, 1996; Borst and Haag, 2001; Lewen et al., 2001; Borst,
2003; Grewe et al., 2003). Here, we demonstrated that the lateral connections between VS cells do increase the width of their receptive fields and hence reduce their spatial resolution. Whether the receptive field widths and coupling strengths between VS cells are under dynamic control is an open question. However, there is likely a trade-off between the spatial acuity of the VS cells and the noise present in their responses. Hence, does the low spatial acuity of these cells hinder the ability of downstream neurons in extracting useful information?

Along these lines, it has been suggested that broad receptive fields in fact aid the extraction of information from a population of neurons. Seung and Sompolinsky (1993) found that the performance of a model that estimates the direction of motion in two dimensions by calculating a population vector from a group of neurons with various orientations is optimal for an intermediate tuning width. The amount of extracted information fell quickly to zero as the tuning width narrowed, compared with the optimum, but fell only slowly as the tuning width increased (Seung and Sompolinsky, 1993). Hence, if the electrical coupling between VS cells increased with decreasing signal-to-noise levels, thus increasing the width of their receptive field, the noise levels within VS cells could be reduced without hindering the ability of downstream neurons to extract relevant flow-field information used for orientation behavior.

\section{References}

Bloomfield S, Völgyi B (2004) Function and plasticity of homologous coupling between AII amacrine cells. Vision Res 44:3297-3306.

Borst A (2003) Noise, not stimulus entropy, determines neural information rate. J Comput Neurosci 14:23-31.

Borst A, Egelhaaf M (1992) In vivo imaging of calcium accumulation in fly interneurons as elicited by visual motion stimulation. Proc Natl Acad Sci USA 89:4139-4143.

Borst A, Haag J (2001) Effects of mean firing on neural information rate. J Comput Neurosci 10:213-221.

de Ruyter van Steveninck R, Bialek W (1995) Reliability and statistical efficiency of a blowfly movement-sensitive neuron. Philos Trans R Soc Lond B Biol Sci 348:321-340.

de Ruyter van Steveninck RR, Laughlin SB (1996) The rate of information transfer at graded-potential synapses. Nature 379:642-645.

DeVries SH, Xiaofeng Q, Robert S, Makous W, Sterling P (2002) Electrical coupling between mammalian cones. Curr Biol 12:1900-1907.

Famiglietti EV, Kolb H (1975) A bistratified amacrine cell and synaptic circuitry in the inner plexiform layer of the retina. Brain Res 84:293-300.

Farrow K, Haag J, Borst A (2003) Input organization of multifunctional motion-sensitive neurons in the blowfly. J Neurosci 23:9805-9811.

Feigenspan A, Teubner B, Willecke K, Weiler R (2001) Expression of neuronal vonnexin36 in AII amacrine cells of the mammalian retina. J Neurosci 21:230-239.

Grewe J, Kretzberg J, Warzecha AK, Egelhaaf M (2003) Impact of photon noise on the reliability of a motion-sensitive neuron in the fly's visual system. J Neurosci 23:10776-10783.

Haag J, Borst A (2002) Dendro-dendritic interactions between motionsensitive large-field neurons in the fly. J Neurosci 22:3227-3233.

Haag J, Borst A (2003) Orientation tuning of motion-sensitive neurons shaped by vertical-horizontal network interactions. J Comp Physiol [A] 189:363-370.

Haag J, Borst A (2004) Neural mechanism underlying complex receptive field properties of motion-sensitive interneurons. Nat Neurosci 7:628-634.

Haag J, Egelhaaf M, Borst A (1992) Dendritic integration of motion information in visual interneurons of the blowfly. Neurosci Lett 140:173-176.

Haag J, Theunissen F, Borst A (1997) The intrinsic electrophysiological characteristics of fly lobula plate tangential cells. II. Active membrane properties. J Comput Neurosci 4:349-369.

Haag J, Denk W, Borst A (2004) Fly motion vision is based on Reichardt 
detectors regardless of the signal-to-noise ratio. Proc Natl Acad Sci USA 101:16333-16338.

Hengstenberg R (1977) Spike response of "non-spiking" visual interneurone. Nature 270:338-340.

Hengstenberg R (1982) Common visual response properties of giant vertical cells in the lobula plate of the blowfly Calliphora. J Comp Physiol [A] 149:179-193.

Hengstenberg R, Hausen K, Hengstenberg B (1982) The number and structure of giant vertical cells (VS) in the lobula plate of the blowfly Calliphora erytrocephala. J Comp Physiol [A] 149:163-177.

Hidaka S, Akahori Y, Kurosawa Y (2004) Dendrodendritic electrical synapses between mammalian retinal ganglion cells. J Neurosci 24:10553-10567.

Jacobs GA, Miller JP (1985) Functional properties of individual neuronal branches isolated in situ by laser photoinactivation. Science 228:344-346.

Kolb H, Jones J (1985) Electron microscopy of Golgi-impregnated photoreceptors reveals connections between red and green cones in the turtle retina. J Neurophysiol 54:304-317.

Krapp HG, Hengstenberg B, Hengstenberg R (1998) Dendritic structure and receptive-field organization of optic flow processing interneurons in the fly. J Neurophysiol 79:1902-1917.

Kurtz R, Warzecha AK, Egelhaaf M (2001) Transfer of visual motion information via graded synapses operates linearly in the natural activity range. J Neurosci 21:6957-6966.

Lamb TD, Simon EJ (1976) The relation between intercellular coupling and electrical noise in turtle photoreceptors. J Physiol (Lond) 263:257-286.

Laughlin SB, Howard J, Blakeslee B (1987) Synaptic limitations to contrast coding in the retina of the blowfly Calliphora. Proc R Soc Lond B Biol Sci 231:437-467.
Lewen GD, Bialek W, de Ruyter van Steveninck RR (2001) Neural coding of naturalistic motion stimuli. Network 12:317-329.

Miller JP, Selverston A (1979) Rapid killing of single neurons by irradiation of intracellularly injected dye. Science 206:702-704.

Owen WG (1985) Chemical and electrical synapses between photoreceptors in the retina of the turtle, Chelydra serpentina. J Comp Neurol 240:423-433.

Raviola E, Gilula NB (1973) Gap junctions between photoreceptor cells in the vertebrate retina. Proc Natl Acad Sci USA 70:1677-1681.

Ribi WA (1978) Gap junctions coupling photoreceptor axons in the first optic ganglion of the fly. Cell Tissue Res 195:299-308.

Seung HS, Sompolinsky H (1993) Simple-models for reading neuronal population codes. Proc Natl Acad Sci USA 90:10749-10753.

Single S, Borst A (1998) Dendritic integration and its role in computing image velocity. Science 281:1848-1850.

Stettoi E, Raviola E, Dacheux RF (1992) Synaptic connections of the narrow-field, bistratified rod amacrine cell (AII) in the rabbit retina. J Comp Neurol 325:152-168.

Tessier-Lavigne M, Attwell D (1988) The effect of photoreceptor coupling and synapse nonlinearity on signal:noise ratio in early visual processing. Proc R Soc Lond B Biol Sci 234:171-197.

Tsukamoto Y, Masarachia P, Schein SJ, Sterling P (1992) Gap junctions between the pedicles of macaque foveal cones. Vision Res 32:1809-1815.

Vaney DI (1991) Many diverse types of retinal neurons show tracer coupling when injected with biocytin or neurobiotin. Neurosci Lett 6:553-562.

Warzecha A-K, Kurtz R, Egelhaaf M (2003) Synaptic transfer of dynamic motion information between identified neurons in the visual system of the blowfly. Neuroscience 119:1103-1112. 